Western University

Scholarship@Western

Human Environments Analysis Lab (HEAL)

$11-2017$

Incorporating the 'Theory of Planned Behavior' into personalized healthcare behavior change research: a call to action

Justine Horne

Janet Madill

Jason A. Gilliland

Follow this and additional works at: https://ir.lib.uwo.ca/healpub 


\title{
Incorporating the 'Theory of Planned Behavior' into personalized healthcare behavior change research: a call to action
}

\author{
Justine Horne*1,2, Janet Madill² \& Jason Gilliland 3,4,5,6 \\ ${ }^{1}$ Faculty of Health Sciences, The University of Western Ontario, London, Canada \\ ${ }^{2}$ Division of Food \& Nutritional Sciences, Brescia University College, The University of Western Ontario, London, Canada \\ ${ }^{3}$ Department of Geography, The University of Western Ontario, London, Canada \\ ${ }^{4}$ Department of Paediatrics, The University of Western Ontario, London, Canada \\ ${ }^{5}$ School of Health Studies, The University of Western Ontario, London, Canada \\ ${ }^{6}$ Department of Epidemiology \& Biostatistics, The University of Western Ontario, London, Canada \\ * Author for correspondence: jhorne5@uwo.ca
}

The 'Theory of Planned Behavior' (TPB) has been tested and validated in the scientific literature across multiple disciplines and is arguably the most widely accepted theory among behavior change academics. Despite this widespread acceptability, the TPB has yet to be incorporated into personalized healthcare behavior change research. Several prominent personalized healthcare researchers suggest that personalizing healthcare recommendations have a positive impact on changes in lifestyle habits. However, research in this area has demonstrated conflicting findings. We provide a scientific and theoretical basis to support a proposed expansion of the TPB to include personalization, and call to action-personalized healthcare behavior change researchers to test this expansion. Specific recommendations for study design are included.

First draft submitted: 25 May 2017; Accepted for publication: 17 August 2017; Published online: 24 November 2017

Keywords: behavior change $\bullet$ genomics $\bullet$ methodology $\bullet$ nutrigenomics $\bullet$ theory

The 'Theory of Reasoned Action', developed in the late 1960s, focused on attitudes and subjective norms as key predictors of human behavior. In 1991, Ajzen published a seminal text in Organizational Behavior and Human Decision Processes where he proposed an important expansion of Ajzen and Fishbein's 'Theory of Reasoned Action' [1]. He coined this new expanded theory, the 'Theory of Planned Behavior' (TPB; see Figure 1) [1].

The TPB posits that there are three main factors contributing to one's intention to perform a behavior, as well as the resulting actual behavior. These three independent factors include: attitudes, subjective norms and perceived behavioral control (Figure 1). The reasoned action approach (RAA) [3] further breaks down these main categories into more descriptive subcategories. Attitudes can be classified as either experiential attitudes or instrumental attitudes. The former refers to affective attitudes such as pleasant-unpleasant, whereas the latter refers to cognitive attitudes such as health-unhealthy [4]. Subjective norms refer to perceived social pressures to perform a behavior, as well as the individual's weighting on the importance of the opinions of others, which leads to behavioral intention through social reward/punishment. This is referred to as the subcategory of injunctive norms. Descriptive norms is the second subcategory for this key construct, and simply refers to the perceived behaviors of others [4]. Perceived behavioral control refers to the perceived extent to which an individual has access to the appropriate resources and opportunities to perform a given behavior and comprises the subcategories of capacity and autonomy. Capacity refers to the perceived ease/difficulty of a given behavior, whereas autonomy refers to one's perception of control over a given behavior [4]. An individual's intention (motivation) to perform a behavior is central to the TPB and can be influenced by these three independent factors and six related subcategories $[1,3,4]$. Overall, the TPB/RAA identifies key proximal determinants of behavior change, which should be considered in intervention studies aimed toward assessing behavior change.

Ajzen's work has been distinguished as having the highest scientific impact score of all Canadian and American social psychology research [5]. Meta-analyses have found that the TPB can be used to predict behaviors from

Future Medicine 


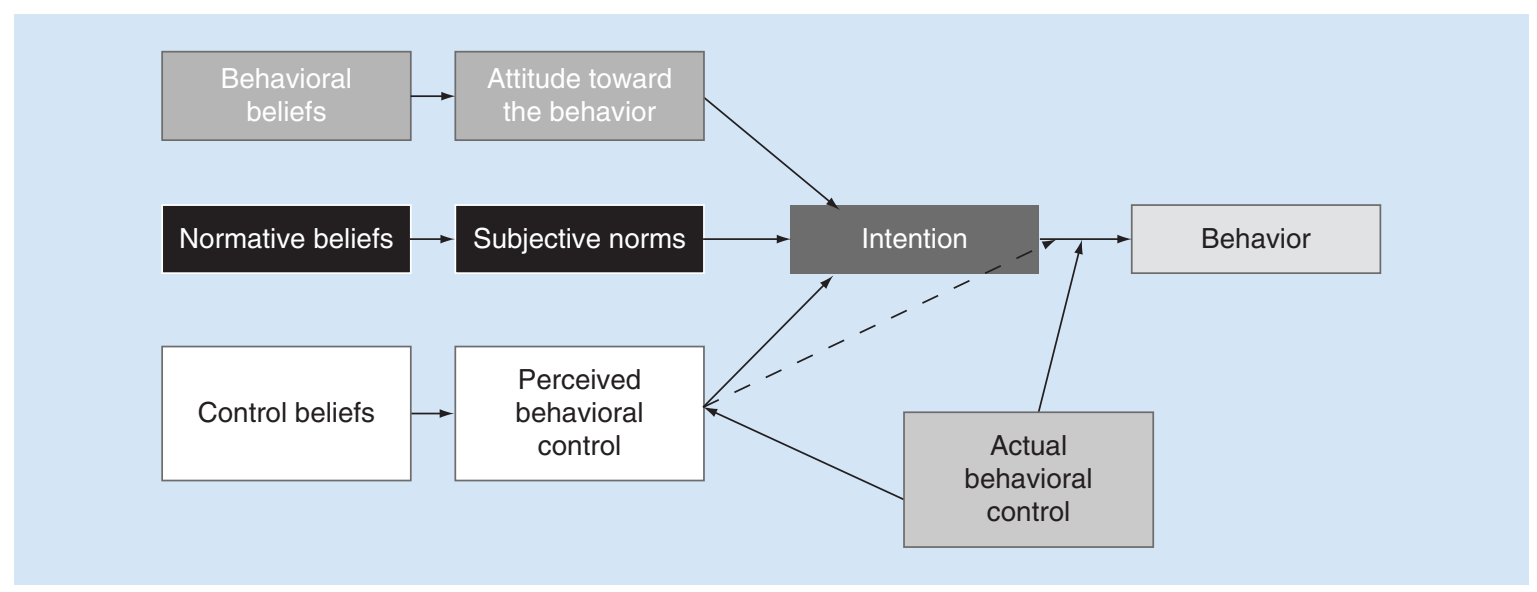

Figure 1. The 'Theory of Planned Behavior'. Solid arrows indicate a direct relationship. The dashed arrow indicates a potential direct relationship, which warrants further investigation.

Adapted with permission from [2].

behavioral intention or perceived behavioral control with mean correlations ranging from 0.4 to 0.53 [6,7]. Metaanalyses have further found that attitudes, subjective norms and/or perceived behavioral control can be used to predict intentions with mean multiple correlations ranging from 0.59 to 0.66 [6-11]. Moreover, with the exception of autonomy, all subcategories of the RAA (outlined above) were found to be significant predictors of Behavioral intention in recently completed regression analyses [4]. Meta-analyses of the TPB in relation to specific healthrelated behaviors including alcohol consumption, diet, sexual health behaviors and treatment adherence in chronic illness have also been recently conducted [12-15]. Notably, the vast majority of these meta-analyses consistently demonstrated medium to large associations between the key constructs of the TPB and behavioral intention as well as actual behavior engagement, with the exception of treatment adherence in chronic illness whereby intentionbehavior effect sizes were small [12-15]. While the TPB has proven to be a strong predictor of behavior change, and is widely used by behavior change researchers, it is clear that there are other factors contributing to behavior change that have not yet been identified and validated within the context of this theory.

With recent advances in personalized healthcare technology, there has been a considerable increase in research pertaining to personalization of healthcare information and recommendations. Personalized healthcare, for the purposes of this paper, refers to healthcare information and recommendations, based on an individual's blood work results and/or individual genetic profile. Several prominent researchers in the field of personalized healthcare suggest that individualizing lifestyle recommendations based on genetics or blood work could have a favorable impact on motivation (behavioral intention) and behavior change [16-18]. To date, changes in several behaviors have been studied in personalized healthcare research including alcohol, nutrition, physical activity, smoking and healthscreening behaviors [16,19-23]. Despite the widespread validation and acceptance of the TPB among academics, the use of this theory in personalized healthcare research is lacking. Notably, a PubMed search of (['Theory of Planned Behavior' AND 'personaliz*' AND 'health'] OR ['Theory of Planned Behavior' AND 'personalis*' AND 'health'] OR ['Theory of Planned Behaviour' AND 'personaliz"' AND 'health'] OR ['Theory of Planned Behaviour' AND 'personalis*' AND 'health']), conducted in April 2017 yielded only two results, neither of which would be considered personalized healthcare research [24,25].

Based on the current state of knowledge pertaining to behavior change and personalized healthcare, this paper calls to action-personalized healthcare behavior change (PHBC) researchers for the incorporation of the TPB into scientific research methods. Furthermore, this paper is the first of its kind to propose a potential expansion of the $\mathrm{TPB}$, based on personalization. It is recommended that this expansion be tested in robust personalized healthcare research to determine if the TPB should be revised to incorporate personalization as a significant predictor of behavioral intention and actual behavior, alongside attitudes, subjective norms and perceived behavioral control. In particular, within the TPB, it is hypothesized that personalization will have a significant impact on attitudes and subjective norms (Figure 2). 


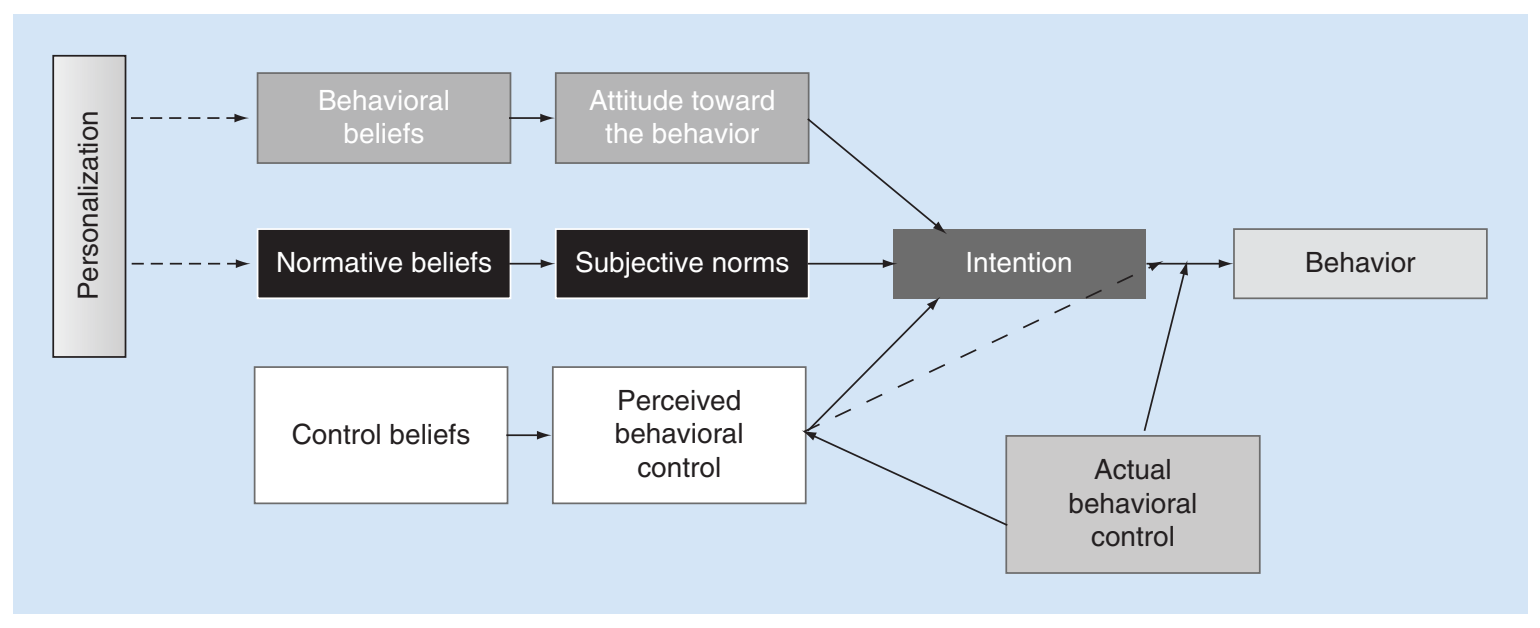

Figure 2. Personalization: a proposed expansion of the 'Theory of Planned Behavior'. Solid arrows indicate a direct relationship. Dashed arrows indicate a potential direct relationship, which warrants further investigation. The present manuscript proposes 'personalization' as a potential future expansion of the 'Theory of Planned Behavior'.

Adapted with permission from [2].

\section{Attitudes toward personalized healthcare}

Prior to delving further into PHBC research conducted to date, it is important to understand the attitudes of healthcare professionals, students in post-secondary health programs and consumers toward personalized healthcare, as these individuals will largely affect the uptake and acceptability of personalized healthcare in society. Most studies assessing attitudes toward personalized healthcare have focused on personalization based on genotyping.

A recent randomized clinical trial found that consumers had favorable attitudes toward participating in genetic testing for personalized healthcare, and those who underwent genetic testing were more likely to recommend it to friends and family [26]. Another recent study found that 'perceived personalization benefit' played a larger role in consumers' intention to utilize personalized nutrition services than 'perceived personalization risk' suggesting that attitudes toward personalized healthcare based on genetics were overall positive [27]. Additionally, a review article concluded that extensive research has demonstrated consumers' keen interest to undergo genetic testing for personalized healthcare [28]. Given these findings, it is not surprising that significant economic growth of genetic testing has been predicted [29].

For healthcare professionals and students in health programs (many of whom will become healthcare professionals), attitudes have been variable with some expressing skepticism and others expressing more positive attitudes toward genetic testing [30-33]. Overall, the availability of genetic testing in clinical practice is growing rapidly [34]. With increasing uptake in clinical practice, the question of whether or not personalized healthcare impacts behavior change is an important one to consider and is proving to be a priority area of research, with at least four review articles published on this topic over the past year alone [35-38].

\section{Current state of PHBC research}

Over the past decade, there has been a considerable amount of research conducted examining the impact of providing personalized healthcare recommendations on motivation and behavior change. One of the largest projects currently underway is the 'Food4Me' project, which commenced its research activities in 2011. 'Food4Me' is a EU funded, large-scale research initiative, which aims to improve scientific knowledge pertaining to personalized healthcare, including motivation and behavior change resulting from the provision of personalized nutrition and physical activity recommendations [39]. We reviewed the 29 peer-reviewed publications posted on the 'Food $4 \mathrm{Me'}$ website [40] and found eight unique articles pertaining to the impact of personalized nutrition and physical activity recommendations on one or more of the following components of the TPB: attitudes and behavior [27,41-46]. However, none of the eight manuscripts specifically referred to the TPB, therefore, it is possible that one or more components of the TPB were included unintentionally. No study from the 'Food4Me' project was designed based upon the TPB specifically. 
Despite the lack of consideration of the TPB in the 'Food4Me' project and other personalized healthcare research projects assessing behavior change, several components of the TPB can be found within PHBC research methods. Of the 29 articles published on the 'Food4Me' website, five studies assessed attitudes related to genetic testing and personalized healthcare, which tended to be positive [27,42,44-46]. Three 'Food4Me' studies analyzed behavior change, and each article found no significant impact on behavior change with the provision of personalized healthcare reports and/or recommendations $[41,43]$. While these studies did not find an impact on behavior change, a recent randomized controlled trial (RCT) found significantly greater reductions in sodium intake when individuals were provided with personalized nutrition advice based on genetics, in comparison to those who were provided with population-based health recommendations [16]. Similarly, a study assessing changes in lifestyle following a geneticbased hypertension intervention also found significant changes in sodium intake among participants provided with DNA-based advice [47]. Furthermore, an RCT found that the provision of personalized nutrition advice enhances motivation (behavioral intention) to change lifestyle behaviors [16].

The inconsistent findings of PHBC research suggest that there are confounding factors influencing behavior change, which are not being considered in the scientific methods. This is likely due to the minimal use of validated theoretical underpinnings to inform study design. While the current body of knowledge appears to have unintentionally addressed one or more components of the TPB, a more comprehensive and intentional approach to the incorporation of the TPB in study design and methodology is required. This would allow for an improved understanding of the extent to which personalized healthcare recommendations, based on genetics and/or blood work, may affect behavioral intention and actual behavior performance. Studies assessing motivation and behavior change should include an analysis of attitudes, subjective norms, perceived behavioral control and actual behavioral control to determine how these factors may alter study outcomes.

\section{A call to action for PHBC research}

It is well known that nutrition, physical activity and wellness strategies can be used to improve health and well-being and decrease the risk for chronic disease but despite this knowledge, rates of obesity and chronic disease continue to climb [48]. Behavior change (or lack thereof) is a key contributor to the increasing rates of obesity and chronic disease despite our increased knowledge of methods to improve health through lifestyle modification [48]. As such, innovative strategies are needed to enhance both intention to change lifestyle habits as well as actual change in lifestyle habits, and personalized healthcare is garnering considerable attention as an innovative healthcare strategy to help combat current global health crises. This paper calls to action PHBC researchers to test the potential expansion of the TPB to include 'personalization' as a possible novel component of the TPB (Figure 2). We propose that personalization may have a significant impact on attitudes and normative beliefs and therefore has the potential to significantly influence behaviors.

In order to incorporate the TPB into personalized healthcare research, it is first important to understand the different components of the theory, including attention to key constructs such as behavioral beliefs, attitude toward the behavior, normative beliefs, subjective norms, control beliefs, perceived behavioral control, actual behavioral control, intention and behavior (Figure 1). In brief, in the TPB, behavioral beliefs are seen to influence attitudes, normative beliefs to influence subjective norms and control beliefs to influence perceived behavioral control. Attitudes, subjective norms and perceived behavioral control all have a significant impact on one's behavioral intention and actual behavior. However, it is also important to note that factors influencing actual behavioral control, such as the social determinants of health [49], strongly predict behaviors regardless of attitudes and subjective norms. Describing each component of the TBP in detail is beyond the scope of this paper and has been accomplished elsewhere [1-3]. Several key resources and seminal texts provide a solid background for deepening understanding of the TPB [1-3].

We contend that this theoretical background could be translated into a more practical application to inform the development of assessment tools with theoretical and practical utility for research in the field. Instructions on completing a TPB questionnaire are available on the University of Massachusetts website [2]. This resource provides a step-by-step guideline to develop a TPB questionnaire, which includes defining the behavior, specifying the research population, formulating items for direct measures and administering a pilot questionnaire. Sample TPB questionnaires are also available on the website and can be used for guidance in the development of assessment tools to be used in the field [2]. Ajzen suggests that multiple regression or structural equation modeling analyses can be used to establish the extent to which attitudes, subjective norms and perceived behavioral control may have 
contributed to intentions. These methods of statistical analyses can also be used to determine the extent to which intentions and perceived behavioral control may have predicted actual behavior(s) [2].

To test the proposed addition of personalization within the TPB, we further advise that an assessment of attitudes toward genetic testing and/or blood work (depending on the method of personalization within the study) be included within the TPB questionnaire. Attitudes toward personalization can be measured on a Likert scale, similar to other questions on Ajzen's sample TPB questionnaire [2]. Ideally, a randomized clinical intervention trial study design could be used for this research, whereby participants are randomly selected to either receive personalized healthcare advice or general population-based healthcare advice. While blinding typically enhances the quality of an RCT, for PHBC research blinding may actually diminish the quality of the results; knowing that one's recommendations are based on their genetics or blood work could influence several aspects of the TPB including behavioral beliefs, attitudes, normative beliefs and subjective norms, thus impacting behavioral intention and behaviors. Therefore, we do not recommend the blinding of participants. Through the use of repeated measures analysis of variance (r-ANOVA) and multiple regression, comparisons can then be made within and between groups to determine the extent to which personalized healthcare advice may have impacted attitudes and subjective norms, and thus behavioral intention as well as actual behavior. To further enhance study design, consideration of the social determinants of health [49] could additionally be incorporated within the study questionnaire to determine if factors such as income, education level, housing and employment, for example, influenced perceived behavioral control and thus behavioral intention and actual behavior.

In addition to testing the proposed expansion of the TPB, several hypotheses could be tested in PHBC research, which incorporate the key constructs of the TPB. Perhaps receiving genetic testing or blood work results could lead to more positive attitudes toward a behavior such as exercising. This may translate into greater intentions to participate in physical activity and actual engagement in physical activity. Or rather, perhaps personalized healthcare only has a significant impact on behavior change in those with a baseline negative attitude toward the behavior of interest; personalized healthcare may significantly alter attitudes and thus lead to greater behavior change but only in those with baseline negative attitudes. These hypotheses have yet to be tested in PHBC research, and should be tested in future studies to advance our understanding of determinants of behavior change in relation to personalized healthcare.

Furthermore, it would be beneficial to conduct a systematic review of PHBC research with a focus on assessing how the (likely unintentional) incorporation of components of the TPB may have impacted the findings of studies conducted to date. While several reviews have been published on the topic of behavior change resulting from personalized healthcare, no review has evaluated studies within the context of the TPB [35-38]. Based on the information presented in this paper, it is evident that there is a need for researchers in the field of personalized healthcare and behavior change to incorporate the TPB into their work as a key theoretical underpinning of study design. Past research in other disciplines can be used to guide research methods [9,12-15].

\section{Conclusion}

The lack of consideration of validated theory in the design of studies assessing PHBC can have a significant impact on the results, as these studies fail to consider key factors that have been shown to affect behavior change. This paper calls to action PHBC researchers to incorporate the TPB in their methods in order to provide a more accurate and thorough assessment of whether or not personalized healthcare advice, based on genetics and/or blood work, has a significant impact on behavior change. This paper suggests that the next expansion of Ajzen's TPB may be the addition of personalization (Figure 2). Future research should seek to test the addition of personalization within the TPB through robust research methods such as RCTs. This call to action is timely in light of the increased focus on innovative healthcare strategies to address the myriad of health concerns arising globally, whereby interventions facilitating behavior change could have a significant impact on global health.

\section{Future perspective}

Based on the current body of knowledge, in addition to the authors' clinical and academic experience, we predict that testing the proposed expansion of the TPB will yield positive findings toward personalization of healthcare recommendations significantly impacting behavior change within some limits. While we predict that personalization will significantly influence behavior change, the ability to change one's behavior must still remain within the individual's actual and perceived behavioral control. In addition, we predict that the method of communicating genetic information will play into one's likelihood of changing, whereby the use of gain-framed messages and actionable 
advice may have a more favorable impact on one's likelihood of altering their lifestyle habits. Comprehension of the results of personalized healthcare testing and recommendations will also play into likelihood of behavior change. As an example, the results of a nutrigenomics test may inform an individual that they have an increased risk for cardiovascular disease, but by limiting caffeine intake this elevated risk could be reduced. This personalized genetic result and consequent gain-framed, actionable recommendation will likely alter one's attitudes toward changing the behavior. If the individual was to inform their family and/or friends about the results of the genetic test, it is likely that they would feel pressure from their social circle to abide by the recommendation. While personalization of healthcare will likely impact attitudes and subjective norms, it is unlikely that it will be a strong enough force to impact perceived and actual behavioral control. If the pressures of work and home life do not allow for adequate sleep and lead to increased stress, one may continue to consume a high quantity of caffeine, regardless of their genetic test. Thus, perceived and actual behavioral controls remain unchanged. It is for this reason that Figure 2 depicts an influence of personalization on attitudes and subjective norms, but not perceived behavioral control. Considering the above-mentioned points, if the individual was sleeping adequately and consumed caffeine for the simple enjoyment of the taste of the caffeinated beverages, the results of the genetic test would likely motivate them to stop drinking caffeinated beverages.

It is further predicted based on clinical experience and our review of the literature that specific aspects of personalized healthcare interventions facilitate behavior change to a greater extent than others. For example, providing genetic testing through a trained healthcare professional rather than using direct-to-consumer methods will likely facilitate behavior change to a greater extent. Moreover, providing actionable recommendations rather than disease risk estimates is likely to result in greater behavior change. We posit that future research will be able to identify similar factors in PHBC research that have been shown to facilitate behavior change and will use this knowledge to design an algorithm for effective personalized healthcare results and recommendations. To our knowledge, this hypothesis has yet to be tested in scientific research.

Overall, we predict that personalization of healthcare will be added to the TPB as a key factor influencing attitudes and subjective norms and thus intention and behavior. Achieving behavior change when it comes to lifestyle habits is arguably one of the most challenging aspects of clinical practice. We further predict that the field of genetic testing will continue to grow as more robust PHBC research is conducted and published. These predictions stem from clinical and academic experience, our review of the literature and theoretical perspectives.

Executive summary

The 'Theory of Planned Behavior'

- The 'Theory of Planned Behavior' (TPB) is a widely accepted and validated behavior change theory, which suggests that there are three main factors contributing to behavior change: attitudes, subjective norms and behavioral control (perceived and actual).

Consideration of theory in personalized healthcare behavior change research

- To date, consideration of theory (especially the TPB) is limited in personalized healthcare behavior change (PHBC) research.

- This lack of consideration of theory helps to explain the heterogeneity of current PHBC research findings.

Predictions for the future

- We predict that robust research will demonstrate that personalization will significantly influence behavior change and thus personalization will be added to the TPB.

- The impact of personalization on behavior change will be limited to a significant influence on attitudes and subjective norms.

Call to action

- This paper calls to action PHBC researchers to test the proposed expansion of the TPB to include personalization.

Conclusion

- Present research demonstrates a lack of consideration of theoretical underpinnings to inform study design, yet the results of several articles demonstrate that personalization is likely a key component to be added of behavior change theory.

- Future research should seek to inform study design using the TPB and determine the extent to which personalization influences key components of the TPB. 


\section{Acknowledgements}

The authors would like to acknowledge Elizabeth Anne Kinsella for her comments during the manuscript-editing process.

Financial \& competing interests disclosure

The authors have no relevant affiliations or financial involvement with any organization or entity with a financial interest in or financial conflict with the subject matter or materials discussed in the manuscript. This includes employment, consultancies, honoraria, stock ownership or options, expert testimony, grants or patents received or pending, or royalties.

No writing assistance was utilized in the production of this manuscript.

\section{References}

Papers of special note have been highlighted as: $\bullet$ of interest; $\bullet \bullet$ of considerable interest

1. Ajzen I. The theory of planned behavior. Orgnizational Behav. Hum. Decis. Process 50, 179-211 (1991).

-. This seminal text provides a thorough overview of the 'Theory of Planned Behavior' (TPB).

2. Ajzen I. Icek Ajzen: theory of planned behavior (2006). http://people.umass.edu/aizen/tpb.html

3. Fishbein M, Ajzen I. Predicting and Changing Behavior: The Reasoned Action Approach. Taylor \& Francis Group Ltd, NY, USA (2010).

4. Mceachan R, Hons BA, Taylor N, Gardner P, Conner M. Meta-analysis of the reasoned action approach (RAA) to understanding health behaviors. Ann. Behav. Med. 50(4), 592-612 (2016).

-• A recently published meta-analysis of the reasoned action approach subcomponents and their effect on intention and behavior.

5. Ajzen I. The theory of planned behaviour: reactions and reflections. Psychol. Health 26(9), 1113-1127 (2011).

-• A detailed review of limitations and opportunities of the TPB, including a presentation of the results of meta-analyses and the theory's application in the health domain.

6. Rivis A, Sheeran P. Descriptive norms as an additional predictor in the theory of planned behaviour: a meta-analysis. Curr. Psychol. 22(3), 218-233 (2003).

7. Armitage CJ, Conner M. Efficacy of the theory of planned behaviour: a meta-analytic review. Br. J. Soc. Psychol. 40(Pt 4), 471-499 (2001).

8. Cheung SF, Chan D. The role of perceived behavioral control in predicting human behavior: a meta-analytic review of studies on the theory of planned behavior. Chinese University Hong Kong (2000) (Unpublished).

9. McEachan RRC, Conner M, Taylor NJ, Lawton RJ. Prospective prediction of health-related behaviours with the theory of planned behaviour: a meta-analysis. Health Psychol. Rev. 5(2), 97-144 (2011).

10. Notani A. Moderators of perceived behavioral control's predictiveness in the theory of planned behavior: a meta-analysis. J. Consum. Psychol. 7(3), 247-271 (1998).

11. Schulze R, Wittmann W. A meta-analysis of the theory of reasoned action and the theory of planned behavior: the principle of compatibility and multidimensionality of beliefs as moderators. In: Meta-Analysis: New Developments and Applications in Medical and Social Sciences. Schulze R. Holling H. Bohning D (Eds). Hogrefe \& Huber Ltd., WA, USA, 219-250 (2003).

12. Cooke R, Dahdah M, Norman P, French DP. How well does the theory of planned behaviour predict alcohol consumption? A systematic review and meta-analysis. Health. Psychol. Rev. 10(2), 148-167 (2016).

13. Andrew BJ, Mullan BA, Wit D et al. Does the theory of planned behaviour explain condom use behaviour among men who have sex with men? A meta-analytic review of the literature. AIDS Behav. 20(12), 2834-2844 (2016).

14. Rich A, Brandes K, Mullan B, Hagger MS, Rich A. Theory of planned behavior and adherence in chronic illness: a meta-analysis. J. Behav. Med. 38(4), 673-688 (2015).

15. Mcdermott MS, Oliver M, Simnadis T et al. The theory of planned behaviour and dietary patterns: a systematic review and meta-analysis. Prev. Med. 81, 150-156 (2015).

16. Nielsen DE, El-Sohemy A. Disclosure of genetic information and change in dietary intake: a randomized controlled trial. PLoS ONE 9(11), e112665 (2014).

17. Nielsen DE, Shih S, El-Sohemy A. Perceptions of genetic testing for personalized nutrition: a randomized trial of DNA-based dietary advice. J. Nutrigenet. Nutrigenomics 7(2), 94-104 (2014).

18. Celis-Morales C, Livingstone KM, Marsaux CFM et al. Design and baseline characteristics of the Food4Me study: a web-based randomised controlled trial of personalised nutrition in seven European countries. Genes Nutr. 10(1), 450 (2015).

19. Hendershot CS, Otto JM, Collins SE, Liang T, Wall TL. Evaluation of a brief web-based genetic feedback intervention for reducing alcohol-related health risks associated with ALDH2. Ann. Behav. Med. 40(1), 77-88 (2010).

20. Marsaux CFM, Celis-Morales C, Livingstone $\mathrm{KM}$ et al. Changes in physical activity following a genetic-based internet-delivered personalized intervention: randomized controlled trial (Food4Me). J. Med. Internet Res. 18(2), 1-15 (2016). 
21. Hishida A, Terazawa T, Mamiya T et al. Efficacy of genotype notification to Japanese smokers on smoking cessation: an intervention study at workplace. Cancer Epidemiol. 34(1), 96-100 (2010).

22. Bloss CS, Schork NJ, Topol EJ. Effect of direct-to-consumer genomewide profiling to assess disease risk. N. Engl. J. Med. 364(6), 524-534 (2011).

23. Roke $\mathrm{K}$, Walton $\mathrm{K}$, Klingel $\mathrm{S}$ et al. Evaluating changes in omega-3 fatty acid intake after receiving personal FADS1 genetic information: a randomized nutrigenetic intervention. Nutrients 9(3), 240 (2017).

24. Middlemass J, Davy Z, Cavanagh K et al. Integrating online communities and social networks with computerised treatment for insomnia. Br. J. Gen. Pract. 62(605), E840-E850 (2012).

25. Denison FC, Reader M, Health F et al. Physical activity in pregnant women with class III obesity: a qualitative exploration of attitudes and behaviours. Midwifery 31(12), 1163-1167 (2015).

26. Kattel S, Onyekwelu T, Brown S-A, Jouni H, Austin E, Kullo IJ. Motivation, perception, and treatment beliefs in the myocardial infarction genes (MI-GENES) randomized clinical trial. J. Genet. Couns. doi:10.1007/s10897-017-0092-9 (2017) (Epub ahead of print).

27. Berezowska A, Fischer ARH, Ronteltap A et al. Understanding consumer evaluations of personalised nutrition services in terms of the privacy calculus: a qualitative study. Public Health Genomics 17(3), 127-140 (2014).

28. Gibney MJ, Walsh MC. The future direction of personalised nutrition: my diet, my phenotype, my genes. Proc. Nutr. Soc. 72(2), 219-225 (2013).

29. Vickery CE, Cotugna N. Incorporating human genetics into dietetics curricula remains a challenge. J. Am. Diet Assoc. 105(4), 583-588 (2005).

30. Bouwman L, Molder H, Hiddink G. Patients, evidence and genes: an exploration of GPs' perspectives on gene-based personalized nutrition advice. Fam. Pract. 25(Suppl. 1), 116-122 (2009).

31. Collins J, Bertrand B, Hayes V et al. The application of genetics and nutritional genomics in practice: an international survey of knowledge, involvement and confidence among dietitians in the US, Australia and the UK. Genes Nutr. 8(6), 523-533 (2013).

32. Cormier H, Tremblay BL, Paradis AM et al. Nutrigenomics - perspectives from registered dietitians: a report from the Quebec-wide e-consultation on nutrigenomics among registered dietitians. J. Hum. Nutr. Diet. 27(4), 391-400 (2014).

33. Horne J, Madill J, O'Connor C. Exploring knowledge and attitudes of personal nutrigenomics testing among dietetic students and its value as a component of dietetic education and practice. Can. J. Clin. Nutr. 4(6), 50-62 (2016).

34. Downie L, Donoghue S, Stutterd C. Advances in genomic testing. AFP 46(4), 200-204 (2017).

35. O’Donovan CB, Walsh MC, Gibney MJ, Brennan L, Gibney ER. Knowing your genes: does this impact behaviour change? Proc. Nutr. Soc. 76(3), 182-191 (2017).

36. Li SX, Ye Z, Whelan K, Truby H. The effect of communicating the genetic risk of cardiometabolic disorders on motivation and actual engagement in preventative lifestyle modification and clinical outcome: a systematic review and meta-analysis of randomised controlled trials. Br. J. Nutr. 116(5), 1-11 (2016).

37. Hollands GJ, French DP, Griffin SJ et al. The impact of communicating genetic risks of disease on risk-reducing health behaviour: systematic review with meta-analysis. BMJ 352, i1102 (2016).

38. French DP, Cameron E, Benton JS, Deaton C, Harvie M. Can communicating personalised disease risk promote healthy behaviour change? A systematic review of systematic reviews. Ann. Behav. Med. 51(5), 718-729 (2017).

- This text provides a current review of personalized medicine (disease risk) behavior change research conducted to date, and includes a brief commentary on lack of consideration of theory in studies conducted thus far.

39. Food4Me. Food4Me: aims and objectives (2011). http://food4me.org/about/aims-and-objectives

40. Food4Me. Food4Me: scientific publications. http://food4me.org/scientific-publications

41. Marsaux CFM, Celis-Morales C, Livingstone KM et al. Changes in physical activity following a genetic-based internet-delivered personalized intervention: randomized controlled trial (Food4Me). J. Med. Internet Res. 18(2), 1-20 (2016).

42. Fallaize R, Macready AL, Butler LT, Ellis JA, Lovegrove JA. An insight into the public acceptance of nutrigenomic-based personalised nutrition. Nutr. Res. Rev. 26(1), 39-48 (2013).

43. Marsaux CFM, Celis-Morales C, Fallaize R et al. Effects of a web-based personalized intervention on physical activity in European adults: a randomized controlled trial. J. Med. Internet Res. 17(10), 1-22 (2015).

44. Poinhos R, Van Der Lans IA, Rankin A et al. Psychological determinants of consumer acceptance of personalised nutrition in 9 European countries. PLoS ONE 9(10), e110614 (2014).

45. Stewart-Knox B, Kuznesof S, Robinson J et al. Factors influencing European consumer uptake of personalised nutrition. Results of a qualitative analysis. Appetite 66, 67-74 (2013).

46. Stewart-Knox BJ, Bunting BP, Gilpin S et al. Attitudes toward genetic testing and personalised nutrition in a representative sample of European consumers. Br. J. Nutr. 101(7), 982-989 (2009).

47. Taylor JY, Wu CY. Effects of genetic counseling for hypertension on changes in lifestyle behaviors among Africa-American women. $J$. Natl Black Nurses Assoc. 20(1), 1-10 (2010). 
48. Arena R, McNeil A, Sagner M, Hills AP. The current global state of key lifestyle characteristics: health and economic implications. Prog. Cardiovasc. Dis. Arena Ross Prog. Cardiovasc. Dis. 59(5), 422-429 (2017).

49. Public Health Agency of Canada. Social determinants of health (2016).

http://cbpp-pcpe.phac-aspc.gc.ca/public-health-topics/social-determinants-of-health/ 